\title{
Dramatic Recovery of a Patient With Wernicke Encephalopathy Presenting With the Lesion of the Corpus Callosum
}

\author{
Halil Onder
}

\begin{abstract}
In this report, I present a rare patient of Wernicke encephalopathy (WE) with a callosal lesion on magnetic resonance imaging at whom a dramatic clinical recovery was achieved by parenteral thiamine therapy. Based on the presentation of this patient, first, I suggest that the awareness of WE among clinicians should be further increased. Second, I re-emphasize the importance of initiation of parenteral thiamine treatments in clinical suspicion of WE which may provide dramatic responses even in patients with atypical lesion sites as in our patient. Future reports including outcomes of a larger number of cases with distinct neuroimaging signs may provide a better understanding of the real clinical burden of the disease as well as unknown aspects of WE.
\end{abstract}

Keywords: Wernicke encephalopathy; Corpus callosum; Parenteral thiamine; Recovery

\section{Introduction}

Wernicke encephalopathy (WE) is a clinical syndrome characterized by the symptoms of mental status changes, ophthalmoplegia, unsteady gait and cerebellar dysfunctions that result from thiamine deficiency [1]. It is associated with significant morbidity and mortality, and, early diagnosis and prompt initiation of thiamine treatment is the critical point for better clinical outcomes [1]. However, the disease is still greatly underdiagnosed in both adults and children [1]. Herein, a rare patient of WE presenting with callosal lesion will be presented. Based on the dramatic recovery of the patient with efficient dosage of intravenous (IV) thiamine treatment, the importance of timely diagnosis and proper diagnosis will be discussed.

\section{Case Report}

A 61-year-old man, with a history of 40 years of alcohol abuse,

Manuscript submitted December 2, 2019, accepted December 16, 2019

Neurology Clinic, Yozgat City Hospital, Yozgat, Turkey.

Email: halilnder@yahoo.com

doi: https://doi.org/10.14740/jnr559 presented with gait impairment, ataxia and progressive amnesia which was recognized over the last 3 months. Progressive cognitive decline, sleep alterations, visual and tactile hallucinations (complaining of stinging and living in the patient's skin) insects and dysarthria were also described during the evolution of the symptomatology which was remarkably recognized during the last 1 month. His condition had worsened significantly during this period, such that he had fall attacks several times due to prominent ataxia and he could not walk without support over the last 3 weeks. Of note, at admission to a health clinic 1 month ago with these symptoms, oral vitamin B-complex supplement (including $10 \mathrm{mg}$ thiamine) $1 \times 1$ was initiated by a general practitioner due to chronic alcohol usage which had not given any benefit. At admission to our clinic, the patient was orientated and cooperative. However, severe dysarthria was recognized. Besides, content and speed of the speech were diminished. He was uninterested in the interview and his emotional responses were weakened that was compatible with apathetic state. The patient could move his eyes in all directions; however, slowness of the movements was recognized (compatible with saccadic dysfunction). Motor and sensory evaluations were within normal limits, whereas deep tendon reflexes were found to be hypoactive. On the other hand, cerebellar examinations revealed severe truncal ataxia. $\mathrm{He}$ could not walk in a straight line. Moreover, his gait was broad-based and significant walking unbalance was present, such that he had even survived the danger of falling several times during the neurological examination. Laboratory examinations including hemogram, biochemistry, thyroid functions and folic acid were normal. B12 level was mildly reduced (150 ng/L (normal range: 180 - 900)). Standardized mini-mental test (SMMT) score was evaluated as 15 points. Cranial magnetic resonance imaging (MRI) showed T2 hyperintense lesions in the splenium and genu corpus callosum, and mamillar body. A slight hyperintensity in the periaqueductal region was also recognized (Fig. 1). Besides, the electroneuromyography study disclosed moderate sensorial axonal polyneuropathy. Taking into account the history of alcohol abuse, the clinical and MRI findings, and the presence of peripheral neuropathy, WE was considered and high dosage IV thiamine treatment was initiated $(3 \times 500 \mathrm{mg}$ IV thiamine in the first 5 days, $1 \times 500 \mathrm{mg}$ in the following 5 days and $100 \mathrm{mg}$ thiamine as maintenance therapy). On evaluation 3 weeks later, a dramatic recovery was achieved. The dysarthric speech was nearly totally recovered and a significant improvement in gait disturbance was provid- 


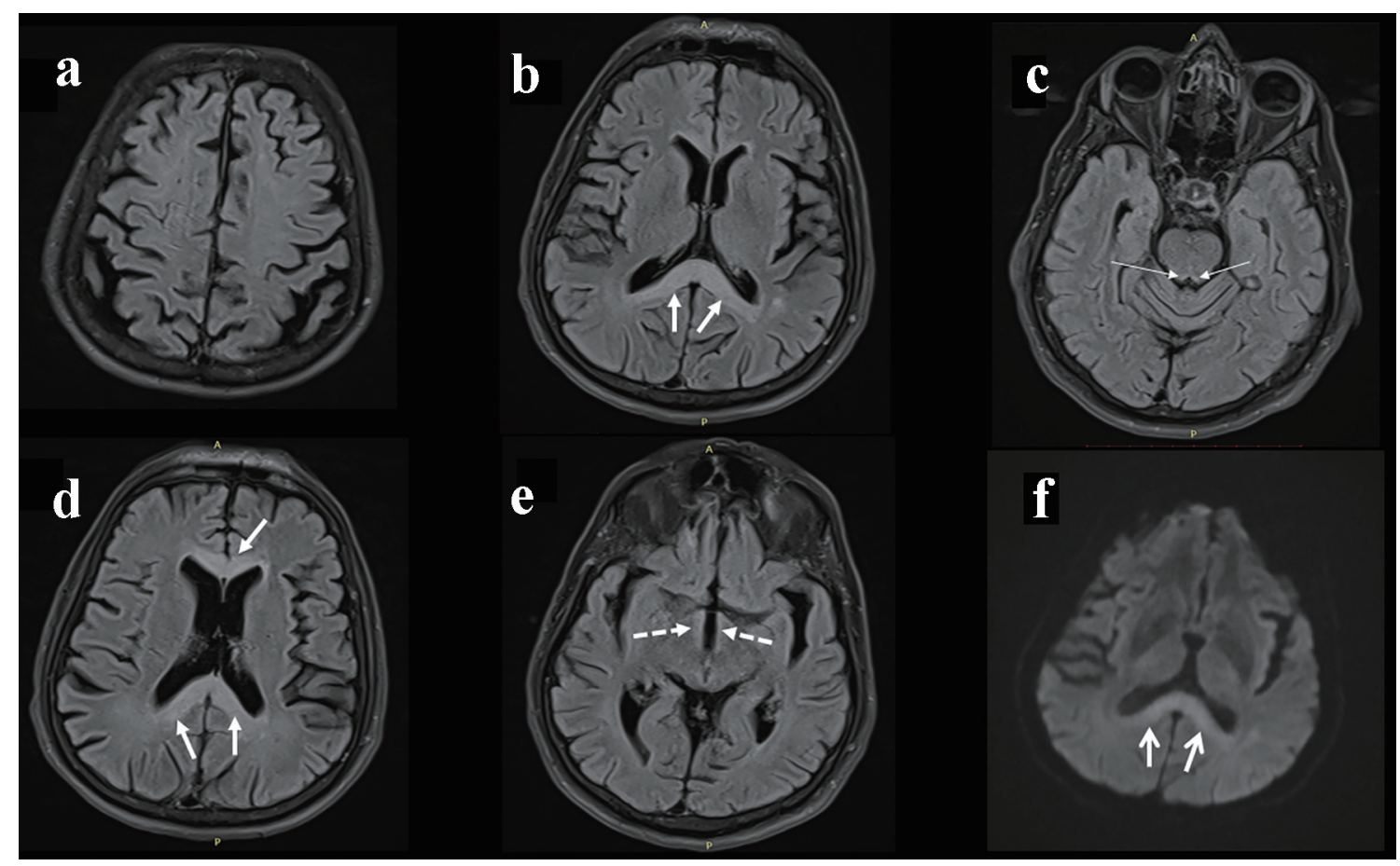

Figure 1. Cranial magnetic resonance image shows T2 hyperintense lesions in the genu and splenium of the corpus callosum (b, d), mamillar body (e). A slight hyperintensity in the periaqueductal region was also recognized (c). Diffusion-weighted imaging shows high signal in the splenial lesion of the corpus callosum (f) (arrows).

ed. He could easily walk without support and perform walking in a straight line. Besides, cognitive symptoms improved significantly. Repeated SMMT examination score was 23 points.

\section{Discussion}

WE is acknowledged to preferentially injure specific brain regions: the dorsomedial thalamus, periaqueductal gray and mamillary bodies [2]. Bilateral symmetric hyperintensity alterations in the periphery of the third ventricle, periaqueductal area, mammillary bodies and midbrain tectal plate on T2weighted MR images have been acknowledged as the characteristic neuroimaging findings of WE [3]. However, less typically, symmetrical signal hyperintensities in additional regions including the caudate nucleus, cranial nerve nuclei, red nuclei, corpus callosum, dentate nuclei and cerebellum have also been reported to be seen in conjunction with WE [4]. In our case, the prominent finding on cranial MRI was T2 hyperintensity in the corpus callosum which is rather an atypical region for WE. In a unique report by Lee et al, the authors remarked to the corpus callosum atrophy in patients with WE and the authors emphasized that the clinical relevance of the corpus callosum atrophy in WE needs to be elucidated in further studies [5]. On the other hand, corpus callosum degeneration has been observed in chronic alcoholics, even in the absence of malnutrition, and has been attributed to cortical and white matter atrophy [6, 7]. Remarkably, in a previous report by Augusto et al, a 52-yearold female patient presenting with hallucinations and cognitive impairment was also reported at whom MRI revealed T2 hyperintense lesions in the corpus callosum that was similar to the lesion in our patient [8]. However, the authors preferred to describe this patient as Marchiafava-Bignami disease, rather than WE. Besides, they have constituted the discussion mainly around symptomatic treatment (rather than thiamine therapy) and the clinical outcome of the patient was not mentioned in detail. Hence, I think that the clinical presentation of our index case may provide substantial perspectives to be kept in mind in the clinical grounds.

Another important point may be that oral vitamin B supplement was also initiated 1 month prior to admission to our clinic which had failed to provide a clinical benefit. However, parenteral thiamine therapy yielded a dramatic improvement in all the symptoms of the patient. Remarkably, the researchers specifically recommend that in clinical suspicion of WE, high dosage parenteral thiamine treatment should be initiated, whereas oral thiamine is inadequate for preventing permanent brain damage [9]. Taken together, I would like to re-emphasize the importance of appropriate dosage and administration route of thiamine in the treatment of WE for optimal clinical responses.

In conclusion, based on the presentation of our patient, first, we suggest that the awareness of WE among clinicians should be further increased. Second, we re-emphasize the importance of initiation of parenteral thiamine treatments in clinical suspicion of WE which may provide dramatic responses even in patients with atypical lesion sites as in our patient. Future reports including outcomes of a larger number of cases with distinct neuroimaging signs may provide a better understanding of the real clinical burden of the disease as well as unknown aspects of WE. 


\section{Acknowledgments}

None to declare.

\section{Financial Disclosure}

None to declare.

\section{Conflict of Interest}

None to declare.

\section{Informed Consent}

Informed consent form has been obtained from the patient and his relatives.

\section{Author Contributions}

The author solely prepared and wrote this article.

\section{References}

1. Sechi G, Serra A. Wernicke's encephalopathy: new clini- cal settings and recent advances in diagnosis and management. Lancet Neurol. 2007;6(5):442-455.

2. Loh Y, Watson WD, Verma A, Krapiva P. Restricted diffusion of the splenium in acute Wernicke's encephalopathy. J Neuroimaging. 2005;15(4):373-375.

3. Jung YC, Chanraud S, Sullivan EV. Neuroimaging of Wernicke's encephalopathy and Korsakoff's syndrome. Neuropsychol Rev. 2012;22(2):170-180.

4. Zuccoli G, Pipitone N. Neuroimaging findings in acute Wernicke's encephalopathy: review of the literature. AJR Am J Roentgenol. 2009;192(2):501-508.

5. Lee ST, Jung YM, Na DL, Park SH, Kim M. Corpus callosum atrophy in Wernicke's encephalopathy. J Neuroimaging. 2005;15(4):367-372.

6. Hommer D, Momenan R, Rawlings R, Ragan P, Williams W, Rio D, Eckardt M. Decreased corpus callosum size among alcoholic women. Arch Neurol. 1996;53(4):359363.

7. Estruch R, Nicolas JM, Salamero M, Aragon C, Sacanella E, Fernandez-Sola J, Urbano-Marquez A. Atrophy of the corpus callosum in chronic alcoholism. J Neurol Sci. 1997;146(2):145-151.

8. Augusto L, Figueiredo R, Costa H, Reis C, Silva ML. Marchiafava-Bignami disease as a cause of visual hallucinations. Braz J Psychiatry. 2015;37(1):82.

9. Flynn A, Macaluso M, D'Empaire I, Troutman MM. Wernicke's encephalopathy: increasing clinician awareness of this serious, Enigmatic, Yet Treatable Disease. The Primary Care Companion For CNS Disorders. 2015; 17. 